\title{
Autoregulation of greA Expression Relies on GraL Rather than on greA Promoter Region
}

\author{
Maciej Dylewski ${ }^{1}$, Llorenç Fernández-Coll ${ }^{2}$, Bożena Bruhn-Olszewska ${ }^{1}$, Carlos Balsalobre ${ }^{2}$ (i) \\ and Katarzyna Potrykus $1, *$ (D) \\ 1 Department of Bacterial Molecular Genetics, Faculty of Biology, University of Gdańsk, W. Stwosza 59, \\ 80-299 Gdańsk, Poland; maciej.dylewski@phdstud.ug.edu.pl (M.D.); bruhn22@gmail.com (B.B.-O.) \\ 2 Department of Genetics, Microbiology and Statistics, Faculty of Biology, University of Barcelona, \\ Av. Diagonal 643, 08028 Barcelona, Spain; llorenc.fernandezcoll@nih.gov (L.F.-C.); cbalsalobre@ub.edu (C.B.) \\ * Correspondence: katarzyna.potrykus@ug.edu.pl; Tel.: +48-585236027
}

Received: 28 September 2019; Accepted: 21 October 2019; Published: 22 October 2019

\begin{abstract}
GreA is a well-characterized transcriptional factor that acts primarily by rescuing stalled RNA polymerase complexes, but has also been shown to be the major transcriptional fidelity and proofreading factor, while it inhibits DNA break repair. Regulation of greA gene expression itself is still not well understood. So far, it has been shown that its expression is driven by two overlapping promoters and that greA leader encodes a small RNA (GraL) that is acting in trans on $n u d E$ mRNA. It has been also shown that GreA autoinhibits its own expression in vivo. Here, we decided to investigate the inner workings of this autoregulatory loop. Transcriptional fusions with lac $Z$ reporter carrying different modifications (made both to the gre A promoter and leader regions) were made to pinpoint the sequences responsible for this autoregulation, while GraL levels were also monitored. Our data indicate that GreA mediated regulation of its own gene expression is dependent on GraL acting in cis (a rare example of dual-action sRNA), rather than on the promoter region. However, a yet unidentified, additional factor seems to participate in this regulation as well. Overall, the GreA/GraL regulatory loop seems to have unique but hard to classify properties.
\end{abstract}

Keywords: greA; GraL; autoregulation; sRNA; RNAP; transcription regulation; gene expression; intrinsic terminator

\section{Introduction}

Gene expression regulation can take place at several levels, e.g., at the transcriptional, post-transcriptional, and translational level. In bacteria, transcriptional regulation usually occurs at the stage of initiation, i.e., activators or repressors bind to a specific promoter region sequence, and enhance or inhibit transcription, respectively. Transcription could also be regulated at the stage of elongation (e.g., by the Escherichia coli GreA and GreB proteins that directly interact with RNA polymerase (RNAP), see below), or at the stage of termination (e.g., by antitermination factors, such as the Nus factors (NusA, NusB, NusG and NusE), which form a complex interaction with both RNAP and specific RNA sequences [1]). Other examples include attenuation mediated by ribosomes or regulation by RNA aptamers bound to RNAP [2-4].

A large group of transcriptional regulators also includes small RNA (sRNA). These short RNA species, ranging in size from 50 to 500 nucleotides (nt) [5], were shown to either act in cis (e.g., antisense RNAs [5] and riboswitches [3]) or in trans, where they enhance or inhibit translation of their targets by diverse mechanisms, relying on complementary base-pairing [6]. Trans acting sRNAs often require a protein chaperone mediating sRNA-target mRNA interaction, called Hfq [5]. 
GreA transcription factor is responsible for rescuing stalled RNAP-DNA complexes that arise as a result of pausing or backtracking, i.e., when polymerase translocates backwards and the $3^{\prime}$ end of the nascent RNA transcript is moved out of register $[7,8]$. Its structure is L-shaped, with the globular C-terminal domain interacting with the $\beta^{\prime}$ subunit rim domains at the entrance to the RNAP secondary channel, and the $N$-terminal finger-like domain being inserted into the secondary channel itself [9]. It is worth mentioning that it is generally accepted that NTP (nucleotide triphosphate) substrates are delivered to the RNAP catalytic center through that channel $[10,11]$. At the very tip of GreA's finger domain are two conserved acidic residues (D41 and E44), necessary to induce the intrinsic endonucleolytic activity of RNAP and thus rescue the stalled complex [9]. It is thought that GreA is not bound to RNAP all the time; in addition, other secondary channel proteins exist, e.g., GreB (a very close GreA homologue), DksA, TraR, or Gfh1 [12-15]. It was shown that there is competition between GreA, GreB, and DksA for binding to RNAP [16-18], as well as that different modifications induced by environmental changes may affect a given secondary channel protein's ability to bind to RNAP (reviewed in [19]).

In addition, GreA was also demonstrated to act at the transcription initiation level, e.g., by enhancing promoter escape [20] or by affecting the RNAP-DNA complex isomerization at the ribosomal $r r n B$ P1 promoter [16]. Interestingly, GreA was recently identified as the major transcriptional proofreading and transcription fidelity factor in Escherichia coli [21,22]. Furthermore, GreA/GreB homologues are widely conserved in other bacteria, and were shown to be necessary for expression of genes related to host invasion (e.g., Salmonella enteritica [23]), and in one case reported to be essential genes in Mycoplasma pneumoniae [24].

Although much is known about GreA's action, especially in E. coli, regulation of its expression is still not fully understood. In our previous work [25], we mapped greA promoters and showed that GreA protein autoinhibits transcription of its own gene in vivo, which was evidenced by monitoring $\beta$-galactosidase activities of the pgre $A$ promoter region-lacZ fusion in either $\triangle g r e A$ background or when GreA was overproduced from a plasmid. In these systems, transcriptional activation (derepression) and transcriptional inhibition were observed, respectively. Still, such a phenomenon was not observed under in vitro transcription conditions in a fully defined system where increasing GreA concentrations were employed. In addition, that study yielded the discovery of GraL, a small RNA encoded in the gre $A$ leader region. We had shown that only about $1 / 3$ of transcripts whose transcription is initiated at the two gre $A$ promoters (P1 and P2) is able to proceed through the intrinsic terminator embedded in the greA's 5' untranslated region (UTR) and form full-length greA mRNA [25]. Interestingly, that transcription termination is highly imprecise, yielding two sets of transcripts: ranging from 49 to $59 \mathrm{nt}$ (transcripts originated from the P1 promoter) and from 37 to $47 \mathrm{nt}$ (P2 promoter originated). The short transcripts were called GraL, and we have recently shown that GraL displays effects as an in trans acting sRNA [26]. A schematic representation of the greA promoter and leader region is presented in Figure 1.

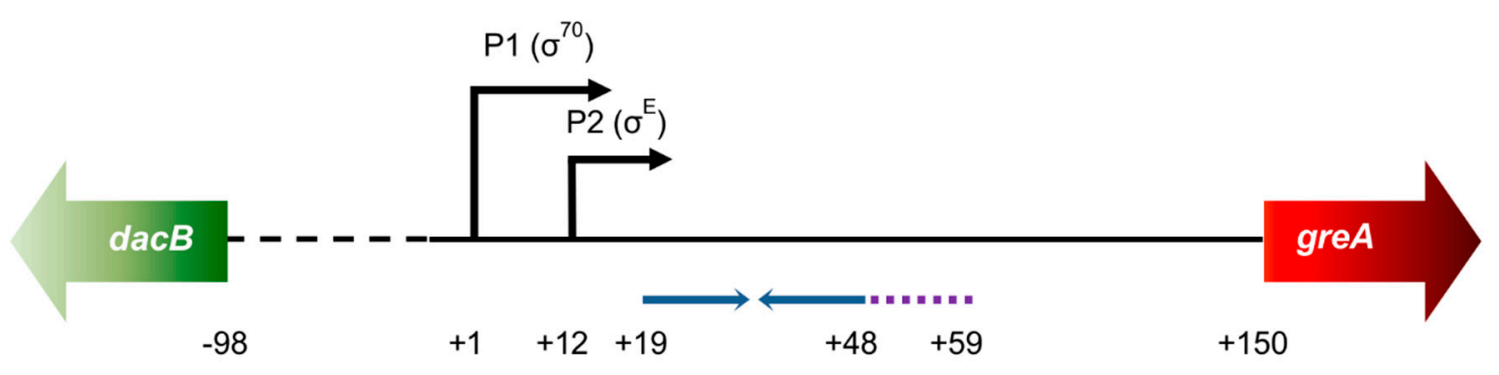

Figure 1. Schematic representation of the greA promoter and leader region. Black arrows indicate P1 ( $\sigma^{70}$-dependent) and P2 ( $\sigma^{\mathrm{E}}$-dependent) promoters. Blue arrows indicate complementary sequences that form the GraL terminator hairpin. Purple dotted line-U-rich sequence where GraL is imprecisely terminated. The first AUG codon of $g r e A$ is at position +150; the first AUG codon of $\operatorname{dacB}$ is at position -98. All numbering is in respect to the P1 transcription start site. Not drawn to scale. 
Here, we decided to dissect the mechanism of GreA mediated inhibition of greA gene expression in vivo by investigating at which stage of transcription this regulation takes place and whether GraL plays any in cis role in this process. We found that GreA autoregulation is promoter region independent, while it relies on the GraL sequence, both upstream of the terminator and forming the terminator hairpin. Thus, GraL is not only an unusual example of imprecise termination but it also seems to represent a new paradigm of an sRNA acting both in cis and in trans. Moreover, feedback inhibition by GreA in vivo is nearly completely abolished by a mutation that eliminates the ability of GreA to cleave backtracked nascent RNA. Although many questions still remain, our data furthers the understanding of how gene expression of the GreA global transcriptional regulator is controlled.

\section{Results}

\subsection{GreA Autoregulation Depends on Catalytically Active GreA}

In the first step of our investigation, we wanted to explore whether the previously observed inhibition of greA expression by GreA depends on the GreA's antipausing activity and ability to induce the intrinsic endonucleolytic activity of RNAP. For this purpose, we used a GreA D41A mutant that has been shown to completely lose its antipause effect on RNAP activity but that is still able to bind to the RNAP secondary channel [12]. A region spanning from -1030 to +175 of gre $A$ (where +1 corresponds to the transcription initiation site of the greA $\mathrm{P} 1$ promoter) was fused to the lacZ reporter gene and introduced onto the chromosome in a single copy. $\beta$-galactosidase activity was monitored in wild-type $(\triangle l a c Z)$ and corresponding $\triangle g r e A$ cells, bearing either a vector control (pBR322), a plasmid encoding wild-type (wt) GreA (pBR-GreA), or its catalytically inactive counterpart (pBR-GreAD41A), cloned under its native promoters.

As shown in Figure 2 and reported previously [25], in the absence of GreA, transcriptional activity of the pgreA-lacZ fusion is significantly increased (about 3.5-fold). Reintroduction of native GreA complemented the $\triangle g r e A$ strain in respect to bringing back the fusion's $\beta$-galactosidase activity to the wt levels. Importantly, under the same conditions, the GreA D41A mutant was unable to repress greA expression, implying not only that the mechanism of GreA autoregulation involves transcription but also that this mechanism is likely to require cleavage of backtracked RNA. This also suggests that GreA autoregulation might take place at a stage other than transcriptional initiation since a double GreA mutant [D41A E44A] was previously shown to retain its regulation at this step of transcription in case of another promoter in vivo [18].

\subsection{GreA Autoregulation is Independent of the Promoter Region}

To verify in another way that GreA autoregulation is independent of the transcription initiation step, several single-copy transcriptional fusions with the lac $Z$ reporter gene were constructed, methodically changing the promoter region elements (Figure 3, left panels). The basic fusion contained a greA region spanning -100 to $+136 \mathrm{bp}$, where +1 again corresponds to the $\mathrm{P} 1$ promoter transcription initiation site (pgre $A_{\text {all }}-$ lac $Z$ ). The gre $A$ promoter region $(-1$ to -36$)$ was then replaced by the plac Uv $_{5}$ promoter, while other elements were retained (plac $\mathrm{Uv}_{5}-\mathrm{greA}-\mathrm{lac} Z$ fusion). In another construct, the region upstream of the promoter was replaced by the plac UP region in addition to the promoter replacement (UP-plac $\mathrm{Uv5}_{5}$-greA-lacZ fusion). In addition, the CRP (catabolite repressor protein) binding site was mutated so as to abolish any possible effects of catabolite repression that regulates plac expression. The changes were at positions $-66(\mathrm{G}->\mathrm{A})$ and $-55(\mathrm{C}->\mathrm{T})$ [27]. Since the P1 and P2 promoters are overlapping, all of the fusions retained part of the -10 sequence of $\mathrm{P} 2$; removing it would alter the GraL sequence. Therefore, as control, a similar transcriptional fusion to UP-plac $\mathrm{UV}_{5}$-greA-lacZ but bearing only the first $10 \mathrm{nt}$ of the gre $A$ leader was also constructed, to exclude the possibility that the $\mathrm{P} 2$ promoter still remained active and was prone to GreA regulation (UP-plac $\mathrm{UV}_{5}-\mathrm{gre} A+10-\mathrm{lacZ}$ ). Importantly, all constructs lack the plac operator region and thus are not under LacI control. The constructs were introduced in a single copy on the chromosome of a $\Delta$ lacZ strain (CF15617), and their activity was 
assessed by $\beta$-galactosidase activity assays in the presence or absence of GreA, overproduced from a low copy plasmid (a pGB2 derivative) under an inducible ptac promoter (pHM1873, called here pGreA; this plasmid does not encode GraL). pHM1883 served as the vector control (pV).

The results are presented in Figure 3 (middle and right panels). It is clear that GreA-mediated inhibition occurs in all three cases where GraL and its downstream region are present (Figure 3a-d), regardless of the promoter region present. The extent of inhibition depends on the presence of isopropyl- $\beta$-D-thio-galactopyranoside (IPTG) —only a minor effect is observed when compared to full downregulation that occurs upon $1 \mathrm{mM}$ IPTG addition (at $\mathrm{OD}_{600} \sim 0.5-, 1.0$ - and 2.0, the fold repression by GreA was calculated to be - for pgreA all-lacZ: 2.5-, 3.7-, and 4.9-fold when compared to the $\mathrm{pV}+\mathrm{IPTG}$ strain (no IPTG: 1.2, 1.1, and 1.2, when compared to the $\mathrm{pV}$ strain); for plac $\mathrm{UV}_{\mathrm{U}}$-greA-lacZ fusion: 2.2-, 3.8- and 4.3-fold (no IPTG: 1.1, 1.2, and 1.6); and for UP-plac $\mathrm{Uv}_{5}-$ greA-lacZ: 2.2-, 2.3-, and 2.8-fold (no IPTG: 1.5, 1.4, and 1.4)). This is probably due to the fact that native gre $A$ was also present on the chromosome and thus overproduction was needed to observe a substantial effect. It should be noted though that addition of IPTG also had a minor effect on the $\mathrm{pV}$ strains.

The only fusion that remained largely unaffected was the control bearing only the +10 gre $A$ leader region (similar $\beta$-galactosidase activities for strains bearing $\mathrm{pGreA}$ and $\mathrm{pV}$ in the presence of IPTG). Altogether, these data independently verify that GreA regulation of greA expression is independent of the promoter region but does depend on the greA leader.

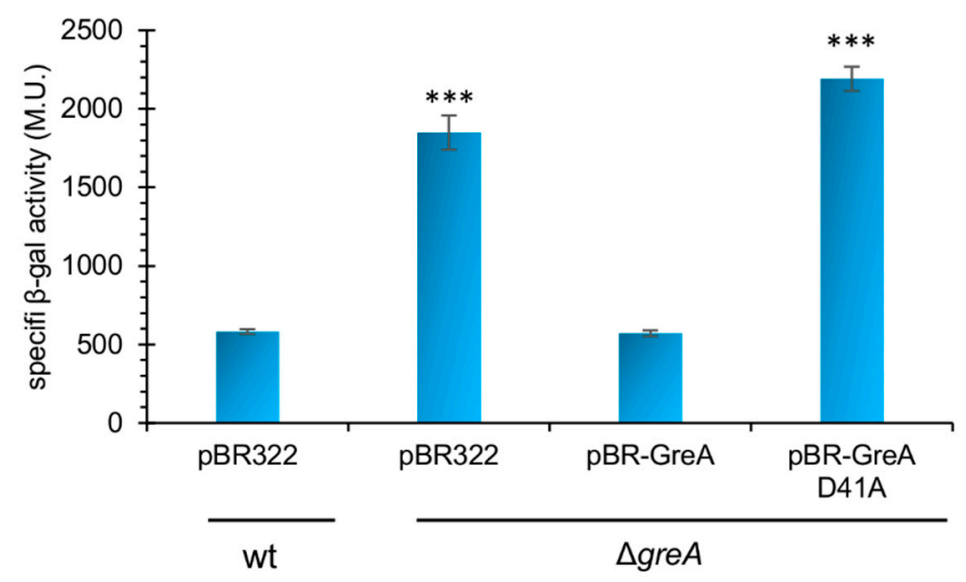

Figure 2. Catalytically active GreA is required for GreA autoregulation. Transcrip tional activity of the gre $A$ promoter and leader region $(-1030$ to +175$)$ was measured by monitoring $\beta$-galactosiadase activity of the pgreA-lacZ fusion in different strain backgrounds: wt-wild type (LFC3-MG1655 $\Delta$ lacZ [17]), $\Delta$ greA (LFC4-MG1655 $\Delta$ lacZ $\Delta g r e A) ;$ pBR-322-vector control; pBR-GreA-vector bearing gre $A$ gene under its native promoter region (i.e., GraL was present as well); pBR-GreAD41- the same as pBR-GreA but encoding catalytically inactive GreA. Cells were grown at $37^{\circ} \mathrm{C}$ in LB (Luria Broth) with aeration; at $\mathrm{OD}_{600} \sim 1.5$ samples were removed and $\beta$-galactosidase activity was measured. Average results from three independent experiments are shown. Error bars represent S.D. For clarity, $p$-values calculated for wt pBR322 vs. $\Delta g r e A$ strains are only shown. ${ }^{* * *} p<0.0005$. 


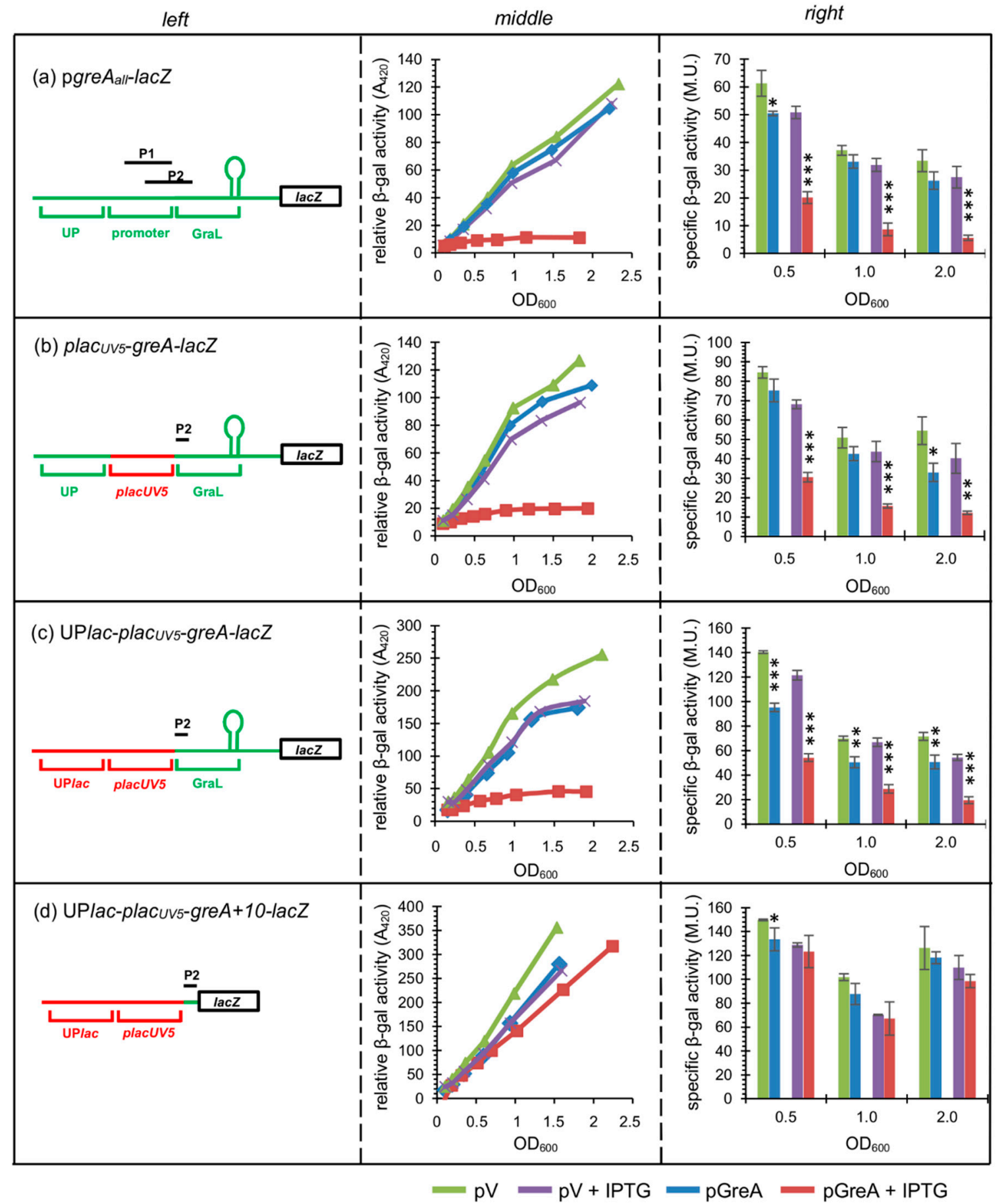

Figure 3. The promoter region does not determine autoregulation exerted by GreA. Assessment of $\beta$-galactosidase activity in strains overproducing GreA from a multicopy plasmid (pGreA) for: (a) pgre $A_{\text {all-lacZ; (b) plac }}$ UV5-greA-lacZ; (c) UP-plac ${ }_{\mathrm{UV} 5}$-greA-lacZ; (d) UP-plac $\mathrm{UV}_{5}$-greA+10-lacZ (negative control) fusions. Wt $\triangle$ lac $Z$ strains carrying appropriate single copy fusions were grown in $\mathrm{LB}$ at $32{ }^{\circ} \mathrm{C}$ with aeration; if present, isopropyl- $\beta$-D-thio-galactopyranoside (IPTG) was added at $\mathrm{OD}_{600} \sim 0.1$ to 1 $\mathrm{mM} ; \mathrm{pV}$ - vector control. Left panels—schematic representation of the lac Z fusions employed; hairpin symbolizes the GraL terminator; not drawn to scale. Middle panels-differential plots of $\beta$-galactosidase activities vs. $\mathrm{OD}_{600}$, following transcriptional activity throughout growth. The slope of each curve corresponds to the specific activity of $\beta$-galactosidase. Right panels-specific $\beta$-galactosidase activities assessed for each strain at $\mathrm{OD}_{600} \sim 0.5,1.0$, and 2.0 (experiment was done in triplicate). Error bars represent S.D. For clarity, $p$-values calculated for $\mathrm{pV}$ vs. $\mathrm{pGreA}$, and $\mathrm{pV}+\mathrm{IPTG}$ vs. $\mathrm{pGreA}+\mathrm{IPTG}$ are only shown. ${ }^{*} p<0.05,{ }^{* *} p<0.005,{ }^{* * *} p<0.0005$. 


\subsection{The Amount of GraL Produced Depends on the GreA Level in the Cell}

Next, we investigated whether the observed GreA-mediated transcriptional inhibition might be due to an increased production of GraL encoded in the greA leader region. As mentioned above, GraL terminates $\sim 2 / 3$ of transcripts that initiate in the pgreA P1P2 promoter region, allowing only $\sim 1 / 3$ of transcripts to extend into full greA mRNA. We reasoned that increased transcriptional termination at the GraL terminator would thus yield less full-length transcripts and cause a decrease in the observed $\beta$-galactosidase activity of the employed fusions.

First, we wanted to establish whether the GraL level is constant throughout the bacterial growth cycle or if it changes. Therefore, GraL levels were monitored by Norther blot analysis, employing the E. coli $\triangle$ lacZ strain (CF15617). As shown in Figure 4, the GraL level is rather constant in the log phase; however, it increases in the stationary phase by 1.8 -fold at the highest $\mathrm{OD}_{600}$ tested, i.e., 3.0, when compared to the GraL level at $\mathrm{OD}_{600}=0.1$.

(a)

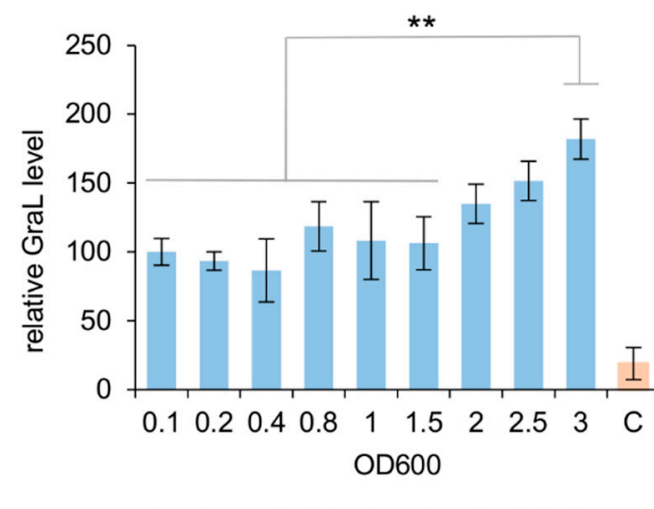

(b)

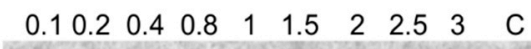

(c)
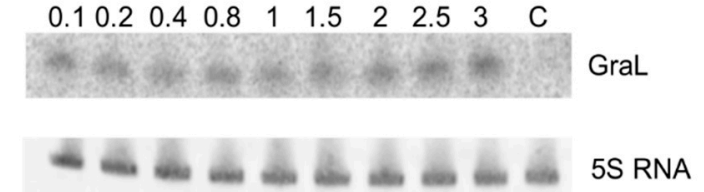

Figure 4. GraL levels monitored throughout bacterial growth. (a) GraL levels established by Northern blot analysis at $\mathrm{OD}_{600}$ 's indicated; data were normalized to 5S RNA levels; the $\triangle$ lacZ $\Delta g r e A \Delta G r a L$ strain (ECMZ1604) served as control, the sample was taken at $\mathrm{OD}_{600} \sim 0.9$ [C-in orange]. Growth of $E$. coli $\Delta$ lacZ (CF15617) was carried out in LB at $32{ }^{\circ} \mathrm{C}$ with aeration. Experiments were done in triplicate. Error bars represent S.D. Calculated $p$-values are indicated (** $p<0.005)$; (b) representative image of Northern blot with GraL visualized; (c) representative image of Northern blot with 5S RNA visualized.

We next asked if the amount of GreA affects GraL abundance. Therefore, we measured the level of GraL produced in the wild type $\Delta l a c Z$ and $\Delta g r e A \Delta l a c Z$ strains in the presence of pGreA or the control plasmid (pV). As demonstrated in Figure 5a, induction of GreA overexpression in wt/pGreA cells by adding $1 \mathrm{mM}$ IPTG moderately increased the GraL level (a $~ 1.25$-fold increase) when compared to IPTG untreated cells or cells carrying vector control, although this increase is not statistically significant. In contrast, in the $\triangle g r e A$ strains carrying $\mathrm{pV}$, the GraL level decreased $\sim 4$-fold when compared to the wild type strain. When pGreA plasmid was now introduced, in the absence of IPTG, the GraL level has increased $\sim 4$-fold, almost reaching the level present in the $\mathrm{wt} / \mathrm{pV}$ cells. When IPTG was added to $1 \mathrm{mM}$ to further induce GreA overexpression, the GraL level in these cells reached the level present in the wt/pA + IPTG cells. These results strongly suggest that increasing the GreA level indeed has a positive effect on GraL production. 
(a)

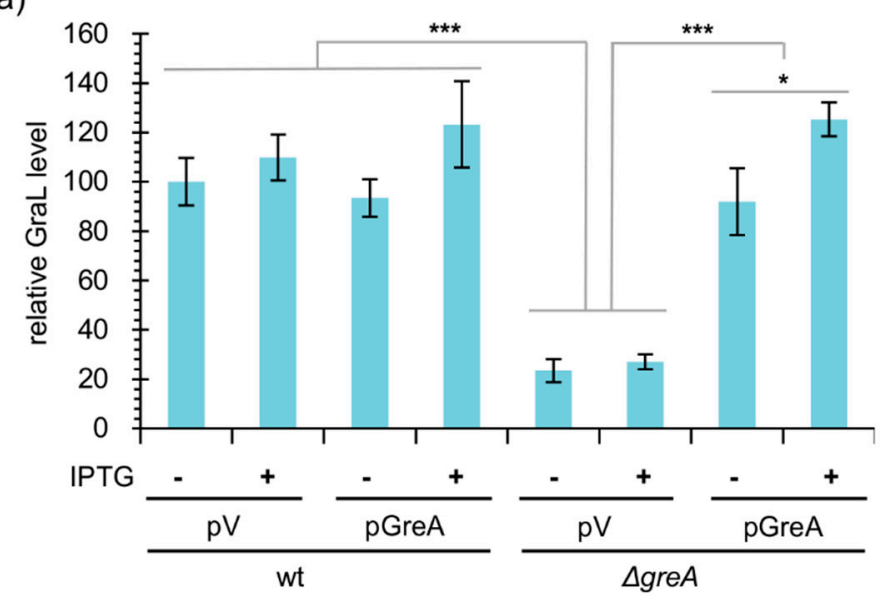

(b)

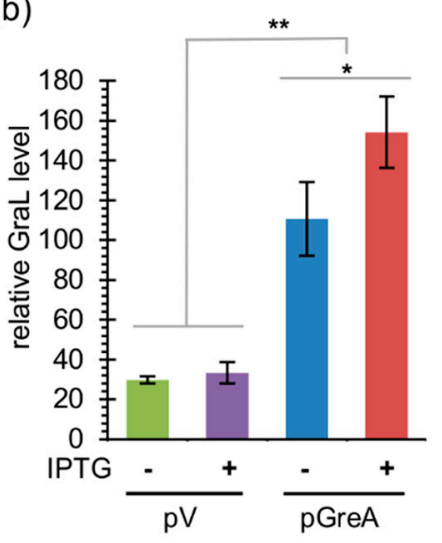

(c)

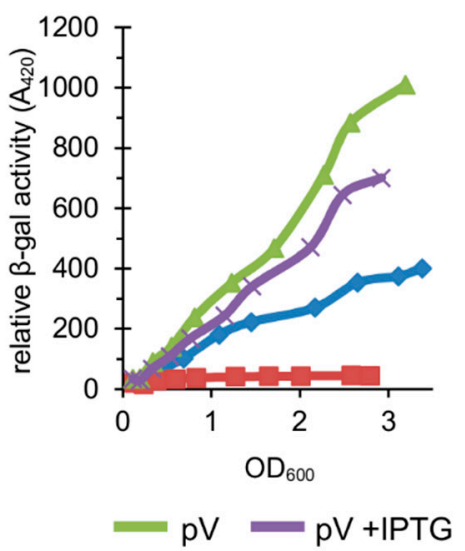

(d)

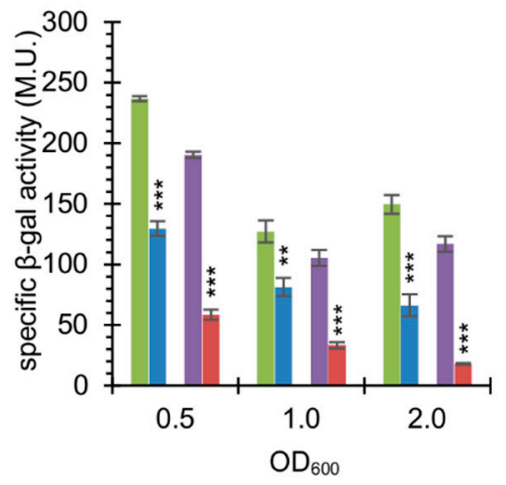

Figure 5. Increased GreA levels correlate with an increase in GraL abundance and a decrease in transcripts that are not terminated at the GraL terminator. (a) Relative GraL level was assessed by Northern blot analysis, as described in Figure 4. If present, IPTG was added to $1 \mathrm{mM}$ at $\mathrm{OD}_{600} \sim 0.1$. Samples were taken from wt or $\triangle g r e A$ strains at $\mathrm{OD}_{600} \sim 3.0$. Data were normalized to $\mathrm{wt} / \mathrm{pV}$; this experiment was done in triplicate. (b) Same as in (a) but with the $\triangle l a c Z \Delta g r e A \triangle G r a L$ strain carrying

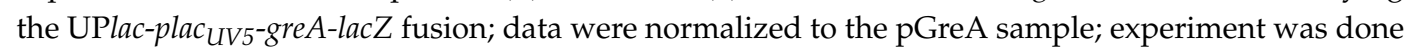
in triplicate. (c) A representative differential plot of $\beta$-galactosidase activities of the strain used in (b). (d) Specific $\beta$-galactosidase activities at $\mathrm{OD}_{600} \sim 0.5,1.0$, and 2.0 of the strains presented in (b) and (c); this experiment was done in triplicate. Error bars represent SD. For clarity, $p$-values calculated only for $\mathrm{pV}$ vs. pGreA, and pV+IPTG vs. pGreA+IPTG are shown in (d). ${ }^{*} p<0.05,{ }^{* *} p<0.005,{ }^{* * *} p<0.0005$.

Next, we wanted to verify that the observed changes in GraL abundance correlate with our previous observations made with promoter region replacement fusions. The single copy UPlac-plac UV5-greA-lacZ $^{-}$ fusion was chosen for these studies. However, this time, the strain was deleted for gre $A$ and GraL on the chromosome (ECMZ1604). In this setting, GraL originates only from the fusion construct under investigation. Figure $5 \mathrm{~b}$ displays the GraL levels assessed by Northern blot analysis, whereas Figure $5 c$,d shows $\beta$-galactosidase reporter activity of the fusion corresponding to the full-length transcripts that were able to proceed through the GraL terminator and yield lacZ mRNA. It is evident that high GraL levels in the strain carrying pGreA plasmid correlate with decreased $\beta$-galactosidase activity of the tested fusion. 


\subsection{The Observed GreA Inhibition of greA Expression is not Due to GraL Acting in trans on its Own mRNA}

Next, we wanted to investigate a possibility that GraL acting in trans, instead of GreA, enhances termination at the GraL terminator and thus increases GraL production. Therefore, similar experiments to those presented in Figure 3 were performed, where $\beta$-galactosidase activity of different lac $Z$ fusions was monitored, but this time GraL (and not GreA) overproduction was induced from a low copy plasmid. No major differences in the activity of the fusions were noted, either in the wt or $\triangle g r e A \Delta G r a L$ background (Figure S1 and Table S1).

In addition, since sRNAs acting in trans often require Hfq protein for binding to their mRNA targets [5], $\beta$-galactosidase activity of the investigated fusions was also monitored in strains either overproducing Hfq or carrying an $h f q$ deletion (again, GreA levels were not altered). The only substantial difference was observed for the $\Delta h f q$ strain carrying the pgreAall-lacZ fusion, whose activity decreased about 1.5-fold in comparison to the wt strain throughout growth (Figure S2 and Table S2). This could imply that another sRNA could perhaps regulate greA transcription at the promoter (either directly or indirectly through another target), but GreA regulation is not involved in this step. When GreA overproduction was induced from the pGreA plasmid in $\Delta h f q$ strains, similar behavior to that reported in Figure 3 was observed for all fusions (Figure S3 and Table S3). We take this to mean that GreA autoregulation does not require Hfq.

\subsection{GreA Mediated Regulation Requires Cis-Acting Gral Sequences in Addition to the Terminator Structure}

The results obtained thus far seem to point to the in cis role of GraL. Alternatively, it could be that not the GraL sequence per se, but rather only the terminator structure participates in the GreA autoregulation, as our previous investigation had hinted that the terminator hairpin might be involved in this process [25]. To answer this question, another set of transcriptional fusions with the lacZ reporter gene were constructed (Figure 6, left panels).

First, the initial GraL sequence $(+1$ to +18$)$ that does not include the terminator hairpin was randomly scrambled, preserving the GC (guanosine and cytosine) content (GraL-Scr-lacZ fusion). In this case, GreA overproduction from the pGreA plasmid still represses the full-length transcript formation as judged from $\beta$-galactosidase reporter activities, although to a much lesser degree (1.2-, 1.4-and 1.7-fold repression at $\mathrm{OD}_{600}=0.5,1.0$, and 2.0, respectively, vs. 2.5, 3.7-, and 4.9-fold inhibition at the respective OD's for the wt fusion, comparing Figure 6a with Figure 3a). Second, we switched the sequences of the terminator hairpin arms (region spanning +19 to +48 ) which retained base pairings to preserve the terminator's secondary structure (Ter-Scr-lacZ fusion, Figure $6 \mathrm{~b}$ ). This resulted in similarly abolished GreA mediated repression in the logarithmic phase of growth, with only some inhibition in the late stationary phase (1.0-, 1.0-, and 1.5-fold inhibition at $\mathrm{OD}_{600}=0.5,1.0$, and 2.0, respectively). Third, a construct combining both modifications was employed ( +1 to +18 scrambled and +19 to +48 region switched; All-Scr-lacZ fusion, Figure $6 c$ ), and yielded similar results (0.9-, $1.0-$, and 1.5-fold repression at $\mathrm{OD}_{600}=0.5,1.0$, and 2.0, respectively). In order to make sure that the scrambled sequences were still functional in termination, in vitro transcription reactions were carried out with these templates and confirmed that indeed termination at the modified sequences still takes place (Figure S4).

Finally, to determine if the sequence downstream of the terminator hairpin might be important for GreA autoregulation, as it may possibly pair with GraL, a fusion devoid of the sequence downstream of the U-rich tail after the terminator was constructed (the region immediately downstream of +59 was deleted; "no downstream sequence": NDS-lacZ). As evidenced in Figure 6d, the downstream sequence does not contribute to GreA autoregulation, since the $\beta$-galactosidase reporter activities were similar to those of the wt fusion (2.8-, 3.9-, and 8.4-fold inhibition at $\mathrm{OD}_{600}=0.5,1.0$, and 2.0, respectively; compare Figure 6d with Figure 3a). 


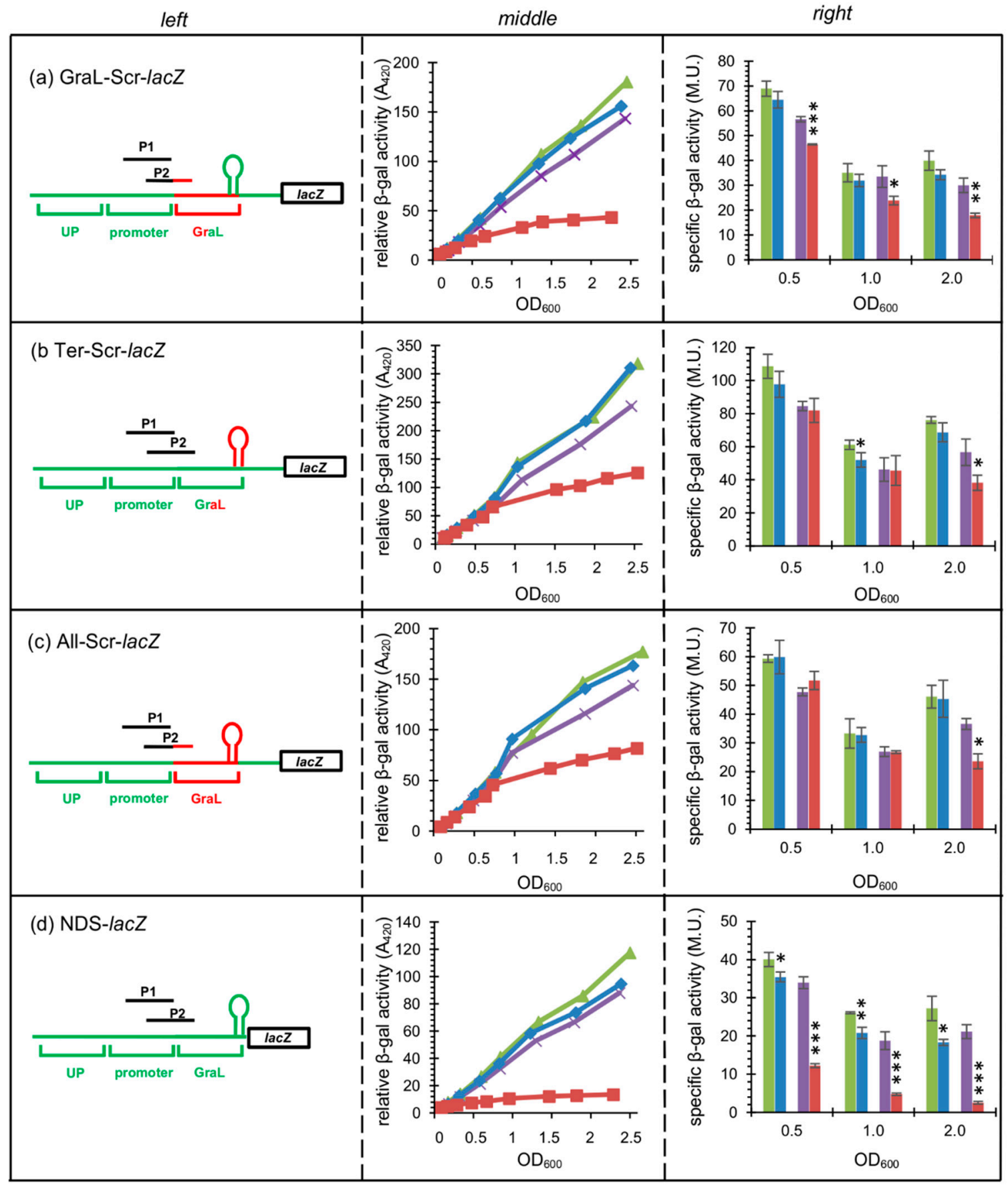

$=p V=p V+I P T G=p G r e A=p G r e A+I P T G$

Figure 6. The GraL sequence (both upstream of and forming the terminator hairpin) determines autoregulation exerted by GreA. Assessment of $\beta$-galactosidase activity in strains overproducing GreA from a multicopy plasmid (pGreA) for: (a) GraL-Scr-lacZ (region from +1 to +18 scrambled); (b) Ter-Scr-lacZ (terminator hairpin arms switched with each other); (c) All-Scr-lacZ ((a) and (b) combined); (d) NDS-lacZ ("no downstream region") fusions. Wt $\Delta$ lacZ (CF15617) strains carrying appropriate single copy fusions were grown in LB at $32{ }^{\circ} \mathrm{C}$ with aeration; if present, IPTG was added at $\mathrm{OD}_{600} \sim 0.1$ to $1 \mathrm{mM} ; \mathrm{pV}$-vector control. Left panels—schematic representation of the lacZ fusions employed; hairpin symbolizes the GraL terminator; not drawn to scale. Middle panels-differential plots of $\beta$-galactosidase activities vs. $\mathrm{OD}_{600}$, following transcriptional activity throughout growth. The slope of each curve corresponds to the specific activity of $\beta$-galactosidase. Right panels-specific $\beta$-galactosidase activities assessed for each strain at $\mathrm{OD}_{600} \sim 0.5,1.0$, and 1.5 (experiment was done in triplicate). Error bars represent S.D. For clarity, $p$-values calculated for $\mathrm{pV}$ vs. $\mathrm{pGreA}$, and $\mathrm{pV}+\mathrm{IPTG} v s$. pGreA+IPTG are only shown. ${ }^{*} p<0.05,{ }^{* *} p<0.005,{ }^{* * *} p<0.0005$. 
All of the above data indicated that it is not only the terminator structure of GraL, but also its sequence (both upstream of the terminator and participating in the terminator formation) that are important for GreA autoregulation. Thus, GraL acting in cis is necessary for GreA autoregulation to occur.

\subsection{None of the Nus Factors nor $\sigma^{E}$ Affect greA Regulation}

Next, we wanted to assess whether Nus factors would have any effect on the GraL terminator read-through since they are known to affect transcriptional elongation, as well as termination and antitermination efficiency $[1,28]$. The four lac $Z$ fusions with promoter region replacements shown in Figure 3a were tested in the absence or presence of plasmids carrying nus $A$, nusB, nusE, or nusG under an IPTG inducible ptac promoter. Wt $\triangle$ lacZ (CF15617) strains were used, expressing greA from the chromosome. No substantial effect was observed when any of the Nus factors were overproduced (Figure S5 and Table S4).

We also tested whether $\sigma^{\mathrm{E}}$ induction would have any effect on the four lac $Z$ fusions tested, since the greA P2 promoter is $\sigma^{\mathrm{E}}$ dependent. We reasoned that GraL termination efficiency might also be under $\sigma^{\mathrm{E}}$ control, since it has been recently suggested that factors inducing a given sRNA transcription often also affect termination at their respective intrinsic terminators [29]. Here, in order to induce $\sigma^{\mathrm{E}}$, we used a pBAI66 plasmid overexpressing a misfolded peptide that accumulates in the periplasm [30]. Again, wt $\triangle$ lacZ (CF15617) strains were used as hosts. Our data indicated that the pgre $A_{\text {all }}$-lacZ fusion is only moderately responsive to $\sigma^{\mathrm{E}}$; however, we observed no differences in $\beta$-galactosidase activity for the other three constructs (Figure S6 and Table S5).

\section{Discussion}

In the study presented here, we turned to GreA, a global transcriptional regulator, shown to have many positive effects, such as rescuing stalled RNAP complexes and being responsible for proofreading and transcriptional fidelity $[9,21,22]$. GreA has been also shown to negatively influence the DNA break repair process [31]. It was also shown to influence gene expression of about 190 genes, when overproduced in E. coli (assuming significant changes in expression are at a log level of 0.5 [32]). On the other hand, competition between GreA and other factors that bind to the RNAP secondary channel is predicted to be perturbed when GreA levels change markedly [18]. One can thus imagine that there is a need for greA expression to be fine-tuned and tightly regulated.

In our earlier work, we showed that gre A leader encodes a small RNA (GraL) and that GreA autoregulates its own gene expression [25]. Here, we demonstrate that this autoregulation depends on GraL acting in cis, i.e., the GraL sequence is necessary and sufficient for the full GreA-mediated repression to occur. Interestingly, the sequence upstream of the terminator is important, as well as the terminator hairpin sequence itself. The promoter region does not seem to play a major part here, indicating that GreA does not affect the transcription initiation step. In addition, catalytically active GreA is required for the autoregulation to take place. One prospect of future research may include investigation of GraL placement in the leader structure, to see if placing it further from the promoters would have any effect on autoregulation.

It has been suggested recently that the genes downstream of the sRNA's terminators are expressed discordantly from the sRNAs encoded in their 5'UTR regions [29]. Given the results described above, this seems not to be the case for GraL and greA. In this instance, GreA levels tightly regulate GraL abundance, and GraL acts in cis to determine the resulting GreA levels. It is not surprising that such a tight regulatory circuit exists since, as mentioned above, GreA is an important transcription regulator and its levels remain fairly constant throughout growth, being slightly higher in the exponential phase of growth than in the stationary phase [33]. What is surprising in this context is that GraL is also acting in trans, with $n u d E$ (encoding a nudix-type hydrolase) being the most likely target [26]. This implies that the GreA regulatory web is even more widespread than previously appreciated. In addition, microarray experiments showed that GraL affects more than 100 genes when overproduced, and it was 
demonstrated that the presence of GraL enhances cellular fitness as well [25]. In addition, another screen revealed about 40 synthetic lethal genes with GraL, representing diverse functions [26].

GraL acting both in cis and in trans is a rare example of a dual action sRNA. To our knowledge, there is only one other well-documented example of this type-SreA riboswitch from Listeria monocytogenes [34], and one report implying such a possibility for Brt1 sRNA from Bartonella hensenae [35]. It is possible that such a dual mechanism of action might be more common than previously thought and more examples await detailed studies.

The simplest model of GreA autoregulation could be imagined to feature catalytically-active GreA as a factor inducing termination at the GraL terminator and thus regulating read-through of the full-length greA mRNA. However, it is intriguing that GreA autoregulation has been only observed so far in vivo and not in vitro ([25]; and Figure S4). This implies that GreA either requires an accessory factor to induce termination (direct mode of GreA autoregulation), or GreA is regulating expression of another factor that in turn regulates GraL termination (indirect mode of GreA autoregulation). Arguments can be made in favor of each model. The involvement of GreA antipause activity (Figure 2) can be seen as evidence of indirect regulation, where GreA could, for example, enhance gene expression of a greA repressor. At the same time, indirect regulation would require the existence of a binding site for such a repressor-in this case, perhaps the GraL sequence could serve as such a site, since it is essential for autoregulation to occur (Figure 3d). However, scrambling of the GraL sequence does not alleviate GreA repression (Figure 6c), and can be seen as evidence against the indirect model. On the other hand, the same arguments can be made in case of the direct model-requirement for GreA antipause activity and specific sequence binding cannot be excluded. Certainly, further studies are required to clarify the exact regulatory mechanism.

As already mentioned, it has been suggested that the efficiency of termination at the sRNA intrinsic terminators is regulated by the same signals (physiological or occurring at times of stress) that induce transcriptional initiation of a given sRNA [29]. This seems not to be true for GreA since here it does not affect the transcription initiation step. However, we wondered whether $\sigma^{\mathrm{E}}$ could be the auxiliary factor enhancing GreA's activity, since $\sigma^{\mathrm{E}}$ controls transcription initiation from the pgreA P2 promoter. Although we did observe a moderate increase in transcriptional activity of the pgre $A_{\text {all }}$-lac $Z$ fusion in vivo when $\sigma^{\mathrm{E}}$ activity was induced by accumulation of misfolded proteins (GreA level was kept constant here), the activity of the other fusions did not increase when the promoter region was replaced with plac ${ }_{U V 5}$. Besides confirming that our basic fusion construct is responsive to $\sigma^{\mathrm{E}}$, this also implies that the auxiliary factor necessary for GreA autoregulation is not under $\sigma^{\mathrm{E}}$ control. We believe that Hfq and the Nus factors are also excluded as the possible accessory factors to GreA. Further studies, perhaps employing transposon random libraries or plasmid libraries of overexpressed genes, are needed to identify the additional factor necessary for GreA autoregulation to occur. Another approach could involve a random point mutant screen for gre $A$ leader isolates that block in vivo autoregulation, which could then be used in a pulldown search for a protein that binds to normal greA leader sequences but not to the autoregulation inefficient mutant. Additionally, another future approach could include investigating a possible role of Rho in this process. Although GraL is very short (only $19 \mathrm{nts}$ before the terminator structure is formed) and terminates at a Rho-independent terminator, it cannot be excluded that the GraL sequence and structure may provide a conditional binding site for Rho, which together with GreA might enhance termination.

How has the GreA/GraL system developed? It seems more plausible that GreA autoregulation (GraL acting in cis) had evolved first, and GraL acting in trans arose as a secondary function. It should be noted that regulation by early transcriptional termination at the $5^{\prime}$ UTR is a common mechanism among bacteria, where attenuators, riboswitches, or T-boxes are employed [36]. All of these mechanisms often rely on alternative RNA secondary structures that under certain conditions may form an intrinsic terminator.

Attempting to qualify GraL as an attenuator or a riboswitch is a complex issue. For one thing, our data show that some remnant GreA autoregulation still persists when either the sequence upstream of 
the terminator or the terminator hairpin sequence is scrambled, pointing to a slight degree of sequence identity independence. On the other hand, even though termination read-through was enhanced in vivo, termination was still observed when using these fusions as templates in in vitro transcription. This indicates that the $\beta$-galactosidase activities observed in vivo did reflect a change in response to the unknown accessory factor, or to GreA and the factor acting together, and did not merely result from abolished termination per se.

On the basis of classic attenuators, one would expect the effector to counteract or alleviate the "normal" (physiological) course of events, i.e., induce terminator formation if usually not formed, or prevent its formation if usually formed. In the case of GreA and GraL, this is not so-GreA (directly or indirectly) seems to amplify termination at the GraL terminator instead of abolishing it. Similar conundrums are encountered when thinking of GraL in terms of a riboswitch-the accessory molecule, acting jointly with GreA, should abolish termination, not enhance it. Of course, it cannot be excluded that under certain conditions GraL termination is overridden-such conditions remain yet to be found. It is also possible that GraL may act as an attenuator or riboswitch under a certain set of conditions, while GreA autoregulation forms a separate or possibly intertwining regulatory circuit.

In the simplest terms, the GreA/GraL system represents a feedback regulatory loop with many unique features. The greA expression is controlled by the GreA protein itself (through GraL acting in cis), but this system also widens GreA's regulatory web (through GraL acting in trans). Definitely, many questions still remain and future studies are needed to dissect the precise mechanism governing greA expression. Our study is an example of how complex and intricate systems are in place to regulate and fine-tune the expression of a global regulator (in this case, a very important transcriptional factor). In addition, the results presented here may aid in the discovery of similar systems, which nevertheless may possess unique variations of their own.

\section{Materials and Methods}

\subsection{Strains and Plasmids}

All transcriptional fusions with lacZ were constructed by introducing an appropriate DNA fragment (obtained either by PCR or through a GeneArt (GeneStrings) service from ThermoFisher Scientific, Waltham, MA, USA) into a pRS415 plasmid (restriction sites: BamHI/EcoRI) [37], except for the gre A -1030 to +175 lac $Z$ fusion, which was constructed by cloning the DNA fragment obtained by PCR (primers G1 and G7) into pRS515 plasmid [37]. Thus, the obtained multicopy fusions were introduced as a single copy on the E. coli chromosome with the use of phage $\lambda$ RS45 and verified to be monolysogens by PCR [38].

For GraL overproduction, pGraL plasmid was constructed by annealing MDGLUP and MDGLDWN primers to each other, followed by 1 cycle of extension by Taq polymerase (GoTaq, Promega, Madison, WI, USA) and subsequent dsDNA purification on Amicon Ultra $0.5 \mathrm{~mL}$ device (10 kD cut-off; Merck, Germany); this fragment was then digested with EcoRI/HindIII and cloned into a pGB2 vector derivative cut with the same enzymes. For GreA overproduction, pHM1873 plasmid (pGreA) was used, and pHM1883 was used as control (pV) [18]. For Nus factor overproduction, pNusA, pNusB, pNusE, and pNusG plasmids were used [39], with pHM1883 as control. For $\sigma^{\mathrm{E}}$ induction, pBAI66 was used, with pTrc99 as control [30,40]. For Hfq overproduction, F+hfq was used, with F+rpoB as control [41]. For the construction of pBR-GreA and pBR-GreA D41A, both alleles were amplified together with their native promoters (using G1 and G11 primers) from MG1655 [42] and TP1204 [43], respectively, and cloned into pBR322 [44] using EcoRI and PstI restriction sites. Gene deletions/modifications were introduced by standard P1 transduction [45]. Strain JW4130-1 was used as a source of $\Delta h f q-722: k a n$ [46]. All strains and plasmids used are listed in Table S6 and Table S7, respectively. The oligonucleotides and synthetic DNA fragments used are listed in Table S8. All oligonucleotides were ordered from Sigma/Merck (Darmstadt, Germany), and all restriction enzymes were purchased from ThermoFisher Scientific (Waltham, MA, USA). 


\section{2. $\beta$-Galactosidase Assays}

These assays were performed as described in [45].

\subsection{Northern Blots}

Northern blot analysis was carried out as described in [25] with modifications that allowed for the detection of chromosome encoded GraL. Briefly, RNA was isolated from cultures of growing cells (5 $\mathrm{OD}_{600}$ units were removed for sampling) with Trizol reagent (ThermoFisher Scientific, Waltham, MA, USA). Next, $20 \mu \mathrm{g}$ of total RNA were loaded onto a 10\% acrylamide TBE-Urea gels (ThermoFisher Scientific, Waltham, MA, USA), and run in $1 \times$ TBE buffer at $160 \mathrm{~V}$ for $58 \mathrm{~min}$. Next, RNA was transferred onto a GeneScreen Plus membrane (Perkin Elmer, Waltham, MA, USA) with the use of Iblot2 Transfer Device (Life Technologies/ThermoFisher Scientific, Waltham, MA, USA) at $20 \mathrm{~V}$ for $3 \mathrm{~min}$. The RNA was then crosslinked to the membrane by $\mathrm{UV}_{254}$ irradiation for $15 \mathrm{~min}$, followed by baking at $80^{\circ} \mathrm{C}$ for $1 \mathrm{~h}$. The membrane was then blocked in UltraHyb-Oligo buffer (ThermoFisher Scientific, Waltham, MA, USA) for $1 \mathrm{~h}$ at $42^{\circ} \mathrm{C}$, followed by the addition of $\mathrm{P}^{32}$-labeled probe to GraL (5'end-labeled pAp2sRNA primer; $1.2 \mu \mathrm{Ci} / \mathrm{mL}$ final concentration), and Cy3 labeled probe to $5 \mathrm{~S}$ RNA (5' end-labeled 5SProbe primer [47]; $50 \mathrm{nM}$ final concentration). The incubation was carried out at $42{ }^{\circ} \mathrm{C}$ for $16 \mathrm{~h}$, and was followed by two $20 \mathrm{~min}$ washes with $2 \times \mathrm{SSC}, 0.5 \%$ SDS buffer at room temperature. The membrane was then directly scanned with the use of Typhoon scanner (GE Healthcare, Life Sciences, Pittsburgh, PA, USA) to visualize 5S RNA (excitation: $532 \mathrm{~nm}$; emission: $580 \mathrm{~nm}$ ), followed by exposition with appropriate Phosphoimager screen to visualize GraL and scanned with the same scanner in the Phosphoimager mode.

\subsection{Single Round in vitro Transcription}

This assay was basically performed as described in [25]. E. coli RNA polymerase was purified as described in [48]. Linear templates were obtained by PCR amplification of the appropriate DNA fragments based on the corresponding fusion constructs with the following primer pair: HMPr368 and pBplasdw (Table S8). GreA was purified as described in [16].

\subsection{Statistical Analysis}

Statistical analysis was carried out either with the use of Microsoft Excel 2013 (Microsoft Corporation, Redmond, WA, USA) or Statistica 13.1 (StatSoft Polska, Cracow, Poland) software. Student's two-tailed t-test was employed for Figures 3, 5d and 6, while one-way Anova with post-hoc Tukey test was used to evaluate data presented in Figures 2, 4 and 5a,b. Differences were deemed as statistically significant for $p<0.05$.

Supplementary Materials: Supplementary materials can be found at http://www.mdpi.com/1422-0067/20/20/ $5224 /$ s1.

Author Contributions: Conceptualization, K.P., and L.F.-C./C.B.; methodology, K.P., M.D., L.F.-C.; validation, K.P., M.D., L.F.-C.; formal analysis, K.P., M.D., L.F.-C.; investigation, M.D., L.F.-C., B.B.-O., and K.P.; resources, K.P., B.B.-O., and C.B.; writing_-original draft preparation, K.P.; writing—review and editing, M.D., L.F.-C., B.B.-O., C.B. and K.P.; visualization, K.P. and M.D.; supervision, K.P. and C.B.; project administration, K.P., and C.B.; funding acquisition, K.P., C.B., M.D., B.B.-O.

Funding: This research was funded by the National Science Centre (Poland), grant number UMO-2013/09/B/NZ1/01066 to K.P.; by the Spanish Ministry of Economy and Competitiveness (grants BIO2010-15417 and PGC2018-096958-B-100) and the Catalan government (grant 2017SGR499) to C.B.; by the FPU grant program (Spanish Ministry of Economy and Competitiveness) to L.F.-C.; and the Funding for Young Researchers, University of Gdańsk [538-L250-B515-17, to M.D; and 538-L250-B538-17, to B.B.-O.]; as well as by statutory funds, University of Gdańsk [DS 530-L250-D694-18].

Acknowledgments: We gratefully acknowledge Mike Cashel for constant support, helpful discussions and critical reading of this manuscript. We also thank Agnieszka Szalewska-Pałasz for encouragement and help with the F factor. We also wish to thank Sylwia Barańska for help with statistical analysis. 
Conflicts of Interest: The authors declare no conflict of interest. The funders had no role in the design of the study; in the collection, analyses, or interpretation of data; in the writing of the manuscript, or in the decision to publish the results.

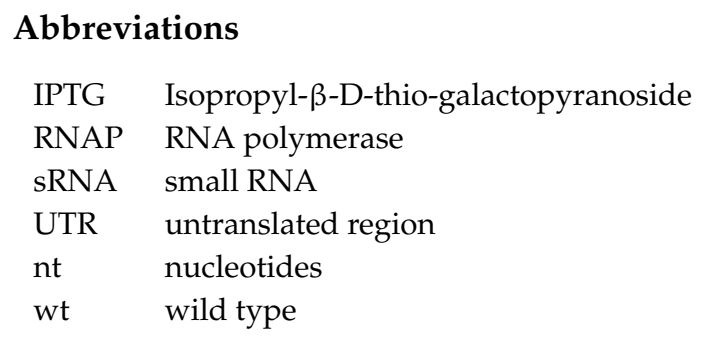

\section{References}

1. Sen, R.; Chalissery, J.; Muteeb, G. Nus Factors of Escherichia coli. EcoSal Plus 2014, 6, 1. [CrossRef] [PubMed]

2. Merino, E.; Yanofsky, C. Transcription attenuation: A highly conserved regulatory strategy used by bacteria. Trends Genet. 2005, 21, 260-264. [CrossRef] [PubMed]

3. McCown, P.J.; Corbino, K.A.; Stav, S.; Sherlock, M.E.; Breaker, R.R. Riboswitch diversity and distribution. RNA 2017, 23, 995-1011. [CrossRef] [PubMed]

4. Sedlyarova, N.; Rescheneder, P.; Magán, A.; Popitsch, N.; Rziha, N.; Bilusic, I.; Epshtein, V.; Zimmermann, B.; Lybecker, M.; Sedlyarov, V.; et al. Natural RNA polymerase aptamers regulate transcription in E. coli. Mol. Cell 2017, 67, 30-43. [CrossRef]

5. Gottesman, S.; Storz, G. Bacterial small RNA regulators: Versatile roles and rapidly evolving variations. Cold Spring Harb. Perspect. Biol. 2011, 3, a003798. [CrossRef]

6. Carrier, M.C.; Lalaouna, D.; Massé, E. Broadening the definition of bacterial small RNAs: Characteristics and mechanisms of action. Annu. Rev. Microbiol. 2018, 72, 141-161. [CrossRef]

7. Komissarova, N.; Kashlev, M. Transcriptional arrest: Escherichia coli RNA polymerase translocates backward, leaving the $3^{\prime}$ end of the RNA intact and extruded. Proc. Natl. Acad. Sci. USA 1997, 94, 1755-1780. [CrossRef]

8. Mustaev, A.; Roberts, J.; Gottesman, M. Transcription elongation. Transcription 2017, 8, 150-161. [CrossRef]

9. Borukhov, S.; Lee, J.; Laptenko, O. Bacterial transcription elongation factors: New insights into molecular mechanism of action. Mol. Microbiol. 2005, 55, 1315-1324. [CrossRef]

10. Zhang, G.; Campbell, E.A.; Minakhin, L.; Richter, C.; Severinov, K.; Darst, S.A. Crystal structure of Thermus aquaticus core RNA polymerase at $3.3 \AA$ resolution. Cell 1999, 98, 811-824. [CrossRef]

11. Vassylyev, D.G.; Sekine, S.; Laptenko, O.; Lee, J.; Vassylyeva, M.N.; Borukhov, S.; Yokoyama, S. Crystal structure of a bacterial RNA polymerase holoenzyme at $2.6 \AA$ resolution. Nature 2002, 417, 712-719. [CrossRef] [PubMed]

12. Laptenko, O.; Lee, J.; Lomakin, I.; Borukhov, S. Transcript cleavage factors GreA and GreB act as a transient catalytic components of RNA polymerase. EMBO J. 2003, 22, 6322-6334. [CrossRef] [PubMed]

13. Nickels, B.E.; Hochschild, A. Regulation of RNA polymerase through the secondary channel. Cell 2004, 118, 281-284. [CrossRef] [PubMed]

14. Blankschein, M.D.; Potrykus, K.; Grace, E.; Choudhary, A.; Vinella, D.; Cashel, M.; Herman, C. TraR, a homolog of a RNAP secondary channel interactor, modulates transcription. PLoS Genet. 2008, 5, e1000345. [CrossRef] [PubMed]

15. Lamour, V.; Hogan, B.P.; Erie, D.A.; Darst, S.A. Crystalstructure of Thermus aquaticus Gfh1, a Gre-factor paralog that inhibits rather than stimulates transcript cleavage. J. Mol. Biol. 2006, 356, 179-188. [CrossRef]

16. Potrykus, K.; Vinella, D.; Murphy, H.; Szalewska-Palasz, A.; D'Ari, R.; Cashel, M. Antagonistic regulation of Escherichia coli ribosomal RNA rrnB P1 promoter activity by GreA and DksA. J. Biol. Chem. 2006, 281, 15238-15248. [CrossRef]

17. Aberg, A.; Shingler, V.; Balsalobre, C. Regulation of the fimB promoter: A case of differential regulation by ppGpp and DksA in vivo. Mol. Microbiol. 2008, 67, 1223-1241. [CrossRef]

18. Vinella, D.; Potrykus, K.; Murphy, H.; Cashel, M. Effects on growth by changes of the balance between GreA, GreB, and DksA suggest mutual competition and functional redundancy in Escherichia coli. J. Bact. 2012, 194, 261-273. [CrossRef] 
19. Fernandez-Coll, L.; Potrykus, K.; Cashel, M. Puzzling conformational changes affecting proteins binding to the RNA polymerase. Proc. Natl. Acad. Sci. USA 2018, 115, 12550-12552. [CrossRef]

20. Hsu, L.M.; Vo, N.V.; Chamberlin, M.J. Escherichia coli transcript cleavage factors GreA and GreB stimulate promoter escape and gene expression in vivo and in vitro. Proc. Natl. Acad. Sci. USA 1995, 92, 11588-11592. [CrossRef]

21. Bubunenko, M.G.; Rattray, A.J.; Gotte, D.R.; Kireeva, M.L.; Irizarry-Caro, J.A.; Li, X.; Jin, D.J.; Court, D.L.; Strathern, J.N.; Kashlev, M. A Cre transcription fidelity reporter identifies GreA as a major RNA proofreading factor in Escherichia coli. Genetics 2017, 206, 179-187. [CrossRef] [PubMed]

22. Traverse, C.C.; Ochman, H. A genome-wide assay specifies only GreA as a transcription fidelity factor in Escherichia coli. G3: Genes Genomes Genet. 2018, 8, 2257-2264. [CrossRef] [PubMed]

23. Gaviria-Cantin, T.; El Mouali, Y.; Le Guyon, S.; Römling, U.; Balsalobre, C. Gre factors-mediated control of hilD transcription is essential for the invasion of epithelial cells by Salmonella enterica serovar Typhimurium. PLoS Pathog. 2017, 13, e1006312. [CrossRef] [PubMed]

24. Hutchison, C.A.; Peterson, S.N.; Gill, S.R.; Cline, R.T.; White, O.; Fraser, C.M.; Smith, H.O.; Venter, J.C. Global transposon mutagenesis and a minimal Mycoplasma genome. Science 1999, 286, 2165-2169. [CrossRef] [PubMed]

25. Potrykus, K.; Murphy, H.; Chen, J.; Epstein, J.; Cashel, M. Imprecise transcription termination within Escherichia coli greA leader gives rise to an array of short transcripts, GraL. Nucleic Acids Res. 2010, 38, 1636-1651. [CrossRef] [PubMed]

26. Dylewski, M.; Ćwiklińska, M.; Potrykus, K. A search for the in trans role of GraL, an Escherichia coli small RNA. Acta Biochim. Pol. 2018, 65, 141-149. [CrossRef]

27. Hirschel, B.J.; Shen, V.; Schlessinger, D. Lactose operon transcription from wild-type and L8-UV5 lac promoters in Escherichia coli treated with chloramphenicol. J. Bact. 1980, 143, 1534-1537.

28. Peters, J.M.; Vangeloff, A.D.; Landick, R. Bacterial transcription terminators: The RNA $3^{\prime}$-end chronicles. J. Mol. Biol. 2011, 412, 793-813. [CrossRef]

29. Chen, J.; Morita, T.; Gottesman, S. Regulation of transcription termination of small RNAs and by small RNAs: Molecular mechanisms and biological functions. Front. Cell. Infect. Microbiol. 2019, 9, 201. [CrossRef]

30. Walsh, N.P.; Alba, B.M.; Bose, B.; Gross, C.A.; Sauer, R.T. OMP peptide signals initiate the envelope-stress response by activating DegS protease via relief of inhibition mediated by its PDZ domain. Cell 2003, 113, 61-71. [CrossRef]

31. Sivaramakrishnan, P.; Sepúlveda, L.A.; Halliday, J.A.; Liu, J.; Núñez, M.A.B.; Golding, I.; Rosenberg, S.M.; Herman, C. The transcription fidelity factor GreA impedes DNA break repair. Nature 2017, 550, $214-218$. [CrossRef] [PubMed]

32. Stepanova, E.; Lee, J.; Ozerova, M.; Semenova, E.; Datsenko, K.; Wanner, B.L.; Severinov, K.; Borukhov, S. Analysis of promoter targets for Escherichia coli transcription elongation factor GreA in vivo and in vitro. J. Bact. 2007, 189, 8772-8785. [CrossRef] [PubMed]

33. Rutherford, S.T.; Lemke, J.J.; Vrentas, C.E.; Gaal, T.; Ross, W.; Gourse, R.L. Effects of DksA, GreA, and GreB on transcription initiation: Insights into the mechanisms of factors that bind in the secondary channel of RNA polymerase. J. Mol. Biol. 2007, 366, 1243-1257. [CrossRef] [PubMed]

34. Loh, E.; Dussurget, O.; Gripenland, J.; Vaitkevicius, K.; Tiensuu, T.; Mandin, P.; Repoila, F.; Buchriesier, C.; Cossart, P.; Johansson, J. A trans-acting riboswitch controls expression of the virulence regulator PrfA in Listeria monocytogenes. Cell 2009, 139, 770-779. [CrossRef] [PubMed]

35. Tu, N.; Carroll, R.K.; Weiss, A.; Shaw, L.N.; Nicolas, G.; Thomas, S.; Lima, A.; Okaro, U.; Anderson, B. A family of genus-specific RNAs in tandem with DNA-binding proteins control expression of the badA major virulence factor gene in Bartonella henselae. Microbiol. Open 2017, 6, e00420. [CrossRef]

36. Naville, M.; Gautheret, D. Premature terminator analysis sheds light on a hidden world of bacterial transcriptional attenuation. Genome Biol. 2010, 11, R97. [CrossRef]

37. Simons, R.W.; Houman, F.; Kleckner, N. Improved single and multicopy lac-based cloning vectors for protein and operon fusions. Gene 1987, 53, 85-96. [CrossRef]

38. Powell, B.S.; Rivas, M.P.; Court, D.L.; Nakamura, Y.; Turnbough, C.L., Jr. Rapid confirmation of single copy lambda prophage integration by PCR. Nucleic Acids Res. 1994, 22, 5765-5766. [CrossRef] 
39. Friedman, D.I.; Olson, E.R.; Johnson, L.L.; Alessi, D.; Craven, M.G. Transcription-dependent competition for a host factor: The function and optimal sequence of the phage lambda box $A$ transcription antitermination signal. Genes Dev. 1990, 4, 2210-2222. [CrossRef]

40. Amann, E.; Ochs, B.; Abel, K.J. Tightly regulated tac promoter vectors useful for the expression of unfused and fused proteins in Escherichia coli. Gene 1988, 69, 301-315. [CrossRef]

41. Saka, K.; Tadenuma, M.; Nakade, S.; Tanaka, N.; Sugawara, H.; Nishikawa, K.; Ichiyoshi, N.; Kitagawa, M.; Mori, H.; Osagawara, N.; et al. A complete set of Escherichia coli open reading frames in mobile plasmids facilitating genetic studies. DNA Res. 2005, 12, 63-68. [CrossRef] [PubMed]

42. Jin, D.J.; Gross, C.A. Mapping and sequencing of mutations in the Escherichia coli rpoB gene that lead to rifampicin resistance. J. Mol. Biol. 1988, 202, 45-58. [CrossRef]

43. Poteete, A.R. Recombination phenotypes of Escherichia coli greA mutants. BMC Mol. Biol. 2011, $12,12$. [CrossRef] [PubMed]

44. Bolivar, F.; Rodriguez, R.L.; Greene, P.J.; Betlach, M.C.; Heyneker, H.L.; Boyer, H.W.; Crosa, J.H.; Falkow, S. Construction and characterization of new cloning vehicles. II. A multipurpose cloning system. Gene 1977, 2, 95-113. [CrossRef]

45. Miller, J.H. Experiments in Molecular Genetics; Cold Spring Harbor Laboratory Press: Cold Spring Harbor, NY, USA, 1972.

46. Baba, T.; Ara, T.; Hasegawa, M.; Takai, Y.; Okumura, Y.; Baba, M.; Datsenko, K.A.; Tomita, M.; Wanner, B.L.; Mori, H. Construction of Escherichia coli K-12 in-frame, single-gene knockout mutants: The Keio collection. Mol. Syst. Biol. 2006, 2, 2006.0008. [CrossRef]

47. Moll, I.; Afonyushkin, T.; Vytvytska, O.; Kaberdin, V.R.; Bläsi, U. Coincident Hfq binding and RNase E cleavage sites on mRNA and small regulatory RNAs. RNA 2003, 9, 1308-1314. [CrossRef]

48. Svetlov, V.; Artsimovitch, I. Purification of bacterial RNA polymerase: Tools and protocols. Methods Mol. Biol. 2015, 1276, 13-29. [CrossRef]

(C) 2019 by the authors. Licensee MDPI, Basel, Switzerland. This article is an open access article distributed under the terms and conditions of the Creative Commons Attribution (CC BY) license (http://creativecommons.org/licenses/by/4.0/). 